\title{
The clinical and biological implications of the focal adhesion kinase pathway in ShenLingLan mediated suppression of cellular migration of ovarian cancer cells
}

\author{
Sioned Owen ${ }^{1,2}$, Fiona Ruge ${ }^{1,2}$, YuNong Gao ${ }^{2}$, Ying Yang ${ }^{3}$, Jianli Hou ${ }^{3}$, Jian Chen ${ }^{3}$, Jane Lane ${ }^{1,2}$, Yong Gao ${ }^{4}$, HongTao Wang ${ }^{4,5}$, Cong Wei,5, \\ Yiling $\mathrm{Wu}^{4,5,6}$ and Wen $\mathrm{G}$ Jiang ${ }^{1,2, *}$ \\ ${ }^{1}$ Cardiff University-Peking University Joint Cancer Institute, Cardiff University School of Medicine, Cardiff, UK \\ 2 Department of Gynaecological Oncology, Peking University Cancer Hospital, Haidian District, Beijing, China \\ ${ }^{3}$ Departments of Gynaecology and Medical Oncology, Yantai Yuhuangding Hospital, Qingdao University, Yantai, Shangdong Province, China \\ ${ }^{4}$ Yiling Medical Research Institute, Shijiazhuang, Hebei Province, China \\ ${ }^{5}$ State Key Laboratory of Collateral Disease Research and Innovation Medicine, Shijiazhuang, Hebei Province, China \\ ${ }^{6}$ Key Disciplines of State Administration of TCM for Collateral Disease, Hebei Province, China
}

\begin{abstract}
The incidence of ovarian cancer in the UK has increased by almost twenty percent since the 1970's and the majority of cases are not diagnosed until the late stages, when metastasis is more likely to have occurred. Focal Adhesion Kinase (FAK) is one of the key protein complexes which is integral to cell migration and has been linked to a variety of solid tumours. ShenLingLan (SLDM) is a traditional herbal medicine which has been formulated for the treatment of solid tumours. This study aimed to establish the impact of SLDM on FAK in ovarian cancer cells in vitro and transcript levels of FAK in an ovarian cancer cohort. FAK and paxillin phosphorylation events stimulated by SLDM treatment were identified using a Kinexus ${ }^{\mathrm{TM}}$ antibody based protein array. The impact of SLDM on cell attachment and migration was evaluated using Electric cell-substrate impedance sensing (ECIS), whilst the changes in focal adhesion complex localisation were assessed using immunofluorescence. In an ovarian cancer cohort, differences in FAK and paxillin transcript levels were assessed against key clinical parameters such as differentiation, stage and survival outcome. SLDM treatment of ovarian cancer cells in vitro resulted in the suppression of FAK and paxillin phosphorylation at several sites, which appeared to manifest as decreased cellular attachment and migration in a range of immortalised ovarian cancer cells. Increased FAK and paxillin transcript copies were observed in high grade and poorly differentiated ovarian tumours as well as in tumours from patients with ovarian cancer related incidence. SLDM has a profound effect on the migratory and adhesive properties of ovarian cancer cells, potentially via inhibitory effects on the activation of the FAK pathway, which is aberrant in clinical ovarian cancers.
\end{abstract}

Keywords: ShenLingLan; ovarian cancer; cell migration; FAK; paxillin; protein array

\section{Introduction}

Cell-matrix adhesion is central to a number of cellular events during cancer development, in particular cell migration and the metastatic spread of cancer cells [1]. Via a range of stimuli, integrins and transmembrane proteins, within normal and malignant cells, interact with extracellular matrix (ECM) proteins and result in cellular movement, as well as other cellular functions [2]. Within cells, integrins are also linked to cytoskeletal proteins which, through phosphorylation events, have the ability to assemble and disassemble focal adhesion complexes [3]. Alterations in these pivotal and dynamic interactions have been identified as key events in the ability of a range of cancer cells to adhere to and disassociate from the ECM, at the primary and secondary sites, essential traits in cellular migration and invasion ultimately contributing to metastatic spread $[4,5]$.
Ovarian cancer annually affects nearly $25 \%$ of the world's female population and is responsible for 140,000 deaths every year [6]. Still renowned for its poor survival rates, the * Corresponding authors: Wen G Jiang, Cardiff China Medical Research
Collaborative, Henry Wellcome Building, School of Medicine, Cardiff
University, Cardiff, CF14 4XN, UK. Email: jiangw@cardiff.ac.uk or Yiling
Wu, Yiling Medical Research Institute, Shijiazhuang, Hebei Province, China.
Email: wuyiling@yiling.cn

Received 21 February 2017 Revised 13 May 2017 Accepted 20 May 2017 Published 30 May 2017

Citation: Owen S, Ruge F, Gao Y, Yang Y, Hou J, Chen J, Lane J, Gao Y, Wang $\mathrm{H}$, Wei C, Yiling Wu, Jiang WG. The clinical and biological implications of the focal adhesion kinase pathway in ShenLingLan mediated suppression of cellular migration of ovarian cancer cells. J Cancer Res Ther. 2017; 5(5):2431. DOI: 10.14312/2052-4994.2017-5

Copyright: (c) 2017 Owen S, et al. Published by NobleResearch Publishers. This is an open-access article distributed under the terms of the Creative Commons Attribution License, which permits unrestricted use, distribution and reproduction in any medium, provided the original author and source are credited. 
worst of all female cancers, the five year survival rate for ovarian cancer patients remains under fifty percent. This poor survival is in part due to the late stages of diagnosis (III and IV) in which up to twenty per cent of patients already have metastatic spread [7]. Evidence suggests that it is tumour recurrence and metastatic spread which are responsible for ovarian cancer related deaths.

Focal Adhesion Kinase (FAK) is a central component within the complexities of matrix-cell interactions. Within the last decade FAK and certain members of the focal adhesion complex proteins (paxillin, alpha-actinin, talin and vinculin) have been found to have aberrant expression levels and/ or levels of activation and thus validated as therapeutic targets, as well as disease progression and prognostic markers in certain solid cancers [8-11]. We have previously reported that a traditional Chinese medicine formula, YangZheng Xiaoji (YZXJ) is able to markedly suppress the phosphorylation levels of FAK in cancer cells and indeed in vascular endothelial cells $[12,13]$. The consequences of blocking FAK using this medicine is of dual benefit, namely the reduction of tumour cell migration as well as the reduction of angiogenesis, which collectively resulted in slower in vivo tumour growth [12, 14-16].

Other kinase pathways, including suppressor of cytokine signalling (SOCS), mitogen activated protein kinases (MAPK) and integrin linked kinase (ILK), lead to transcriptional regulation, resulting in gene expression changes which also participate in the regulation of migration, adhesion and proliferation of cells. A number of specific and nonspecific compounds, some of which have already found their way to the clinics as cancer therapeutic agents, have been shown to be able to suppress the activation of FAK, and hence the motile and aggressive nature of cancer cells [8]. On this basis, we investigated another formulation, ShenLingLan (SLDM), which was modified based on YZXJ, by removal of two ingredients in order to improve the efficacy of the formulation. There are promising early clinical signs that this medicine benefits a variety of cancer patients $[17,18]$. Currently, SLDM is used as a formulated capsule, particularly for those who are receiving chemotherapy. It has been demonstrated that the medicine is safe in clinical trials and was able to influence T-cell functions [19]. We have recently reported that the medicine was able to influence the GSK-3 signalling pathways in ovarian cancer cells $[20,21]$.

The present study aimed to investigate if, and subsequently how, the medicine may work using a series of methods, in a hope to find and eventually provide scientific support for using this medicine in patients. We primarily focused on the action of SLDM on FAK, FAK- mediated cellmatrix adhesion and cellular migration. We particularly concentrated on the effect of the medicine on FAK and members of the focal adhesion complex and indeed the immediate downstream kinases in ovarian cancer cells, a highly aggressive tumour type that has a strong link to FAK activation. We also investigated the expression of FAK and paxillin in a cohort of human ovarian tumours. SLDM had a profound effect on FAK and its downstream signalling events, leading to suppression of cell-matrix adhesion and cellular migration of ovarian cancer cells.

\section{Material and methods}

\section{Materials and cells}

Two ovarian cell lines, SKOV-3 and COV-504, were purchased from LGC Standard/ ATCC (Southampton, England, UK). Both cell lines were cultured in Dulbecco's modified eagle medium (DMEM) (Sigma Aldrich, Dorset, UK) supplemented with $10 \%$ foetal calf serum (12133C, Sigma Aldrich, Dorset, UK) and antibiotic antimycotic solution (A5955, Sigma Aldrich, Dorset, UK) in an incubator pre-set to $37^{\circ} \mathrm{C}$ and $5 \%$ carbon dioxide. PF573228, a small inhibitor for FAK was purchased from Tocris (Bristol, England, UK). Human paxillin antibody (BD610051) was purchased from BD Transduction laboratories (Oxford, England, UK). FITCconjugated secondary antibody (F5262) was purchased from Sigma Aldrich (Dorset, UK).

\section{Tumour samples}

Ovarian tumours ( $n=52$ ) were collected with patient consent immediately after surgery and stored at $-80^{\circ} \mathrm{C}$ until use. Key clinical and pathological information, including tumour staging, differentiation and outcome were also obtained from the clinical database (Table 1). The age of patients ranged from 36 to 73 years of age.

\begin{tabular}{lc} 
Table 1 The clinical and pathological information of the cohort. \\
\hline Clinical information & Patient numbers $(n)$ \\
\hline Stage & 7 \\
$1 / 2$ & 45 \\
$3 / 4$ & \\
Differentiation & 38 \\
Low/non & 8 \\
Moderate & 6 \\
High & \\
Incidence & 25 \\
Incidence free & 27 \\
With incidence & \\
Survival & 36 \\
Alive & 16 \\
Died & \\
\hline
\end{tabular}

\section{ShenLingLan extract (SLDM)}

Fourteen medicinal herbs (including Astragalus, Ligustrum lucidum, ginseng, Ganoderma lucidum, Curcuma, Gynostemma, Atractylodes (fried), Poria, Cordyceps sinensis, Xu Changqing, Eupolyphaga, Panax, diffusa, Scutellaria barbata, The Divine Comedy (fried)) have been combined by Yiling Pharmaceuticals (Shijiazhuang, Hebei, China) to form ShenLingLan. Using a DMSO based method [13] a SLDM extract was prepared from ShenLingLan. In brief, ShenLingLan formulated powder was combined with DMSO in a fixed weight/volume ratio before the mixture was agitated on a rotating wheel (at 100rpm) (Labinco BV, Wolf Laboratory, York, England, UK) for $12 \mathrm{~h}$ at $4^{\circ} \mathrm{C}$. Subsequently the mixture was centrifuged at $15,000 \times$ $g$, for $20 \mathrm{~min}$ at $4^{\circ} \mathrm{C}$. From the fractionated mixture the clear supernatant was collected, pooled and filtered using filtration filters (pore size $0.20 \mu \mathrm{m}$, Sartorius Stedim, Sartorius, Epson, Surrey, UK). This pooled concentrated 
extract was diluted in a balanced salt solution (BSS) and standardised using the extract's optical density, calculated with a spectrophotometer set at a wavelength of $450 \mathrm{~nm}$ (Biotek, Wolf Laboratory). A master preparation which gave $0.25 \mathrm{OD}$ at $450 \mathrm{~nm}$ was stored as the master stock and named SLDM for all subsequent experiments.

\section{Immunofluorescent staining}

Ovarian cancer cells (3-4 × 104/well) were seeded into 8-well Millicell EZ glass slides (Merck Millipore, Cork, Ireland) and left to settle overnight before being treated with SLDM for two hours. Slides were fixed using ice cold ethanol for 30 min prior to being washed with Tris buffered saline (TBS) (25 $\mathrm{mM}, \mathrm{pH}$ 8.4). Subsequently cells were lightly permeabilised with $0.1 \%$ Triton $\mathrm{X} 100$ for $3 \mathrm{~min}$. After thorough washing, slides were blocked with TBS which contained $7.5 \%$ goat serum for at least two hours. Primary paxillin antibody was diluted in the blocking buffer and added to the chamber slides which were left to incubate overnight at $4^{\circ} \mathrm{C}$. Prior to FITC- tagged secondary antibody (1:250) being added for one hour, slides were washed with TBS. Slides were further washed and mounted using FluorSave ${ }^{\mathrm{TM}}$ (Calbiochem, Nottingham, England, UK). Images were captured using an Olympus microscope and a Hamamatsu digital camera.

\section{Protein array}

Ovarian cancer cells were cultured to $90 \%$ confluence in two T75 tissue culture flasks, medium was removed and the cells washed with TBS buffer before being replaced with DMEM supplemented with 5\% FCS. After five hours, SLDM treatment was prepared in DMEM supplemented with 5\% FCS and added to the cells. After five hours the ovarian cancer cells were scraped from the flasks and pelleted by centrifugation at 2,500rpm for $5 \mathrm{~min}$. Lysis buffer was added to the cell pellets and samples placed on a rotating wheel for one hour at $4^{\circ} \mathrm{C}$. This was followed by the lysate samples being centrifuged at $12,000 \mathrm{~g}$ for $10 \mathrm{~min}$ at $4^{\circ} \mathrm{C}$. After centrifugation supernatants from the samples were collected and quantified based on absorbance prior to being adjusted to $2 \mathrm{mg} / \mathrm{ml}$ and stored at $-20^{\circ} \mathrm{C}$ until further use. KAM850 antibody based protein arrays, which capture 854 antibodies spotted on array slides (Kinexus Bioinformatics Ltd, Vancouver, Canada) were used during the course of this study. The following key parameters were collected and used for the subsequent data analyses: Globally Normalized Signal Intensity - globally normalized background corrected intensity values were calculated by combining all the intensities from the net signal median values for each sample. Z Scores - Z score transformation corrected data internally within a single sample. Z Score difference - The difference between the observed protein $Z$ scores in samples for comparison. Z Ratios - Divide the $Z$ score differences by the SD of all the differences for each comparison.

\section{Electric cell-substrate impedance sensing (ECIS)}

This method was used to analyse ovarian cell adhesion and cell migration. Briefly, 96-well W96E1 microarrays were used on an ECIS Ztheta instrument (Applied Biophysics Ltd, Troy, New Jersey, USA) set up in a pre-set incubator $\left(37^{\circ} \mathrm{C}\right.$ and $5 \%$ carbon dioxide). Ovarian cancer cells were added to an array in triplicate and once attached to the instrument were immediately tracked by the software over a range of frequencies $(1,000 \mathrm{~Hz}$ to $64,000 \mathrm{~Hz})$ using automated gold electrodes. To model cellular migration, once the electronic traces had plateaued indicating a complete monolayer had been formed, an electrical wounding (2,000 mA for $20 \mathrm{sec}$ each) was applied through the gold modules creating artificial wounds. Cells were again immediately tracked as they migrated back over the gold electrodes. Both the adhesion and migration were analysed using the mathematical modelling methods as previously described [20, 21].

\section{Quantitative analysis of FAK and paxillin transcripts in tumour samples}

Transcript levels of both FAK and paxillin were quantitatively determined using real-time quantitative PCR performed on Applied Biosicence StepOne Plus (Paisley, England, UK). The quantitative detection was based on a modified Amplifluor ${ }^{\mathrm{TM}}$ probe method previously reported $[20,21]$. Primer details are given in Table 2 including the internal house-keeping control cytokeratin-19 (CK-19) and levels of FAK and paxillin transcripts were generated using an internal standard (PDPL), of known copy number, which was simultaneously amplified with the tumour samples. The reaction conditions were as follows: $94^{\circ} \mathrm{C}$ for $5 \mathrm{~min}, 96$ cycles at $94^{\circ} \mathrm{C}$ for $15 \mathrm{sec}, 55^{\circ} \mathrm{C}$ for $35 \mathrm{sec}$ and $72^{\circ} \mathrm{C}$ for 20 sec. The results are shown in two ways: levels of transcripts based on equivalent amounts of mRNA, and as a target: CK-19 ratio [22].

Table 2 Primer sequences for quantitative PCR

\begin{tabular}{lll}
\hline Primer name & Sense & Anti-sense \\
\hline FAK & CTATCCAGGTCAGGCATCT & $\begin{array}{l}\text { ACTGAACCTGACCGTACAC } \\
\text { GCAGGTCCAATACTGTAGA }\end{array}$ \\
Paxillin & ACAGTCGCCAAAGGAGTC & $\begin{array}{l}\text { ACTGAACCTGACCGTACAA } \\
\text { GTGGGTGCAGACGAAGT }\end{array}$ \\
CK-19 & AGCCACTACTACACGACCAT & $\begin{array}{l}\text { ACTGAACCTGACCGTACAT } \\
\text { CGATCTGCAGGACAATC }\end{array}$ \\
PDPL & GAATCATCGTTGTGGTATG & $\begin{array}{l}\text { ACTGAACCTGACCGTACAC } \\
\text { TTTCATTTGCCTATCACAT }\end{array}$ \\
\hline
\end{tabular}

* ACTGAACCTGACCGTACA - Z sequence for uniprimer probe.

\section{Statistical analysis}

Minitab (Minitab Ltd, Coventry, UK) statistical software package (version 14) was used to perform statistical analysis on tumour RNA samples. Data which was normally distributed was assessed using the two-sample $t$-test, whilst non-normally distributed data was assessed using the Mann-Whitney test. Z score was applied to the Kinexus data where a score of 1.64 or -1.64 was $p=0.05$.

\section{Results}

ShenLingLan showed inhibitory effects on the phosphorylation of FAK, paxillin and the downstream signalling events in ovarian cancer cells

The Kinexus protein array identified a range of phosphorylation sites in which activity was decreased after SKOV-3 ovarian cancer cells had been treated with SLDM (Figure 1). Though there appeared to be little change detected in the pan-specific FAK antibody, dramatic decreases in phosphorylation were detected at 
serine-910 (S-910), tyrosine-397 (Y-397) and the combined sites of tyrosine-576 and -577 (Y-576 + Y-577) compared to the untreated control (Figure 1a). Phosphorylation of paxillin was also decreased in SKOV-3 cells with the most noticeable decrease being observed in the pan-specific antibody compared to the untreated control (Figure 1b). Small decreases in tyrosine-119 (Y-119) and tyrosine-31 (Y-31) phosphorylation were also detected by the Kinexus protein array. Subsequent downstream tyrosine kinase targets for FAK and paxillin were also included in the array (Figure 1c). Of the FAK downstream events, pan-specific
Csk and Shc1 tyrosine-349 (Y-349) and tyrosine-350 (Y-349 + Y-350) phosphorylation events were decreased in SKOV-3 treated cells compared to the untreated control. Treatment of SKOV-3 cells with SLDM appeared to increase the phosphorylation of Rac1, though the activity at serine71 (S-71) appeared to be reduced compared to the untreated control. No significant changes were detected by the pan-specific Src antibody, however a slight increase was detected at Src tyrosine-419. Detection levels of ILK1 and Fyn, a member of the Src family of kinases, showed little difference between the treated and control cells. (a)

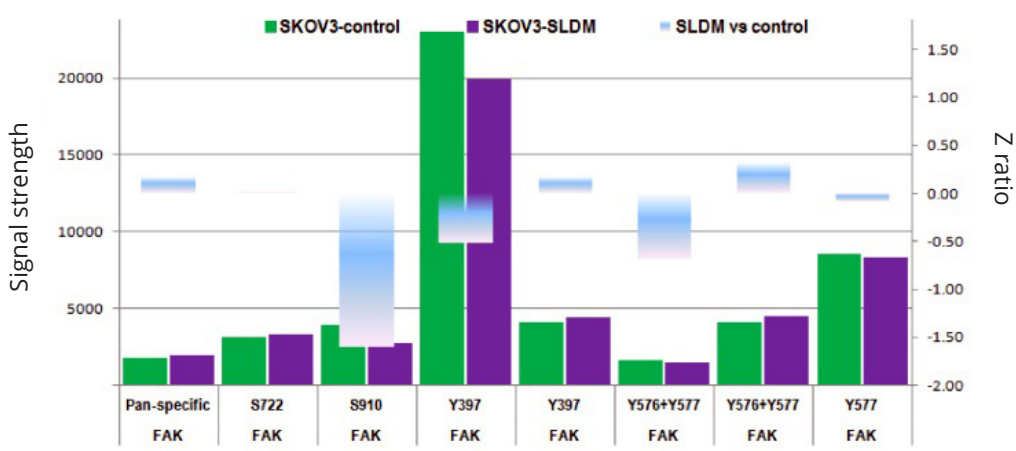

(b)

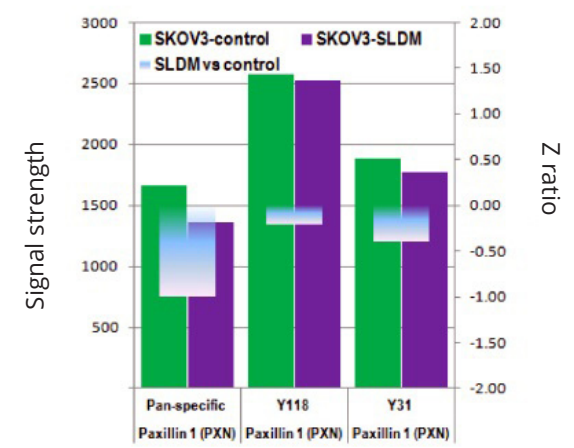

(c)

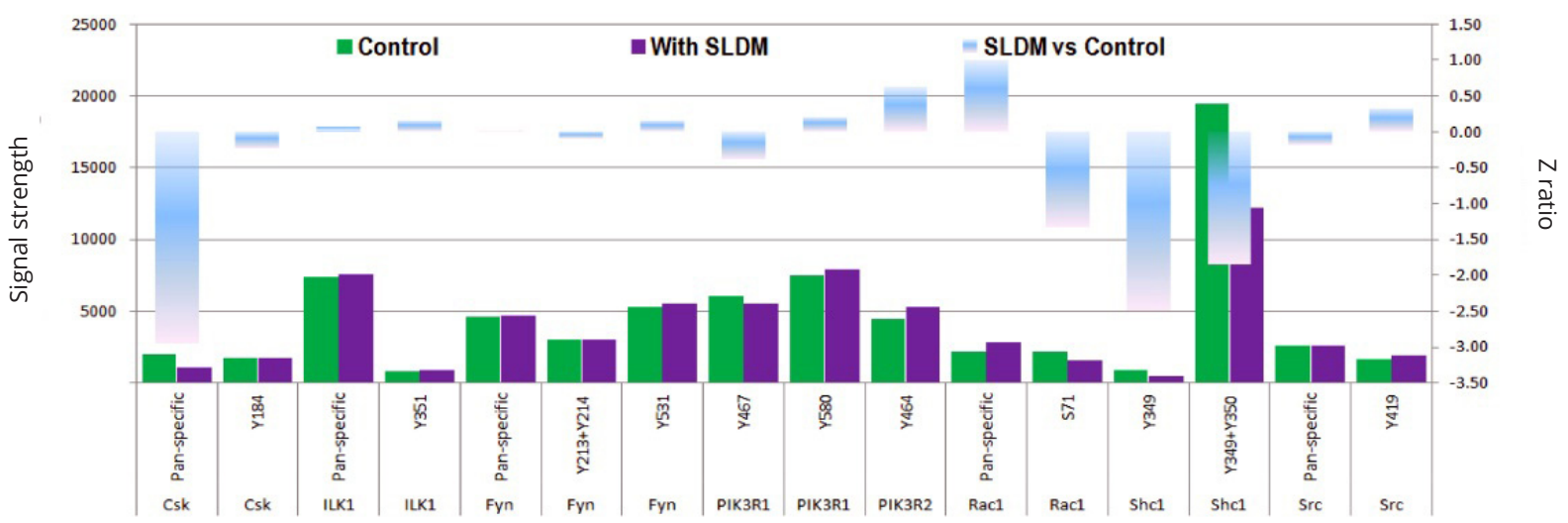

Figure 1 Phosphorylation changes in FAK, paxillin and downstream molecules in SKOV-3 cells after treatment with SLDM.

Signal strength, indicative of expression for pan-specific FAK and specific serine and tyrosine sites with FAK, were screened in SKOV-3 ovarian cancer cells after treatment with SLDM (a). Changes in phosphorylation state was seen at serine-910 (S-910), tyrosine-397 (Y-397) and a combination of tyrosine-576 and 577 (Y-576 + -577) compared to the untreated control, duplication represents use of different antibodies to probe the same phosphorylation site present on the same chip. Reductions in signal strength were also detected at all three of the paxillin sites detected compared to the untreated control (b). Downstream protein kinases were also screened on the antibody array (c). Reductions in signal strength were seen in Csk1 and Shc1, whilst an increase was seen in Rac1. No obvious detection changes were seen in ILK1 and Fyn. Signal strength changes are represented by the green bars for the untreated control and purple bars for the SLDM treated cells. Z-ratio is represented by the blue bar in which the middle is zero and the increase or decrease is represented by the directional change. Average measurement of duplicates shown. Z-ratio >1.64 was considered significant.
ShenLingLan treatment affects paxillin localisation in ovarian cancer cells

Immunofluorescence staining was used to explore the changes in paxillin detected by the Kinexus array, and explore any potential localisation changes which might occur in the ovarian cancer cells (Figure 2). In the untreated SKOV-3 (top panel) cells paxillin appears to be diffuse in the cytoplasm, with some small amounts clustered at the cell membrane potentially indicating focal adhesion clusters (indicated by white arrows). After treatment with SLDM the clusters appear to become more prominent at the cell membrane (white arrows, top right panel). In contrast, in the untreated COV-504 (bottom panel) ovarian cancer cells intense clusters of paxillin staining are clear (white arrows) and appear to be potentially organised along the actin cytoskeleton (bottom left pane, red arrows). COV-504 cells treated with SLDM appear to show a reduced number of these intense paxillin clusters (white arrows, bottom right panel) with some cells demonstrating diffuse cytoplasmic staining (red arrows). 

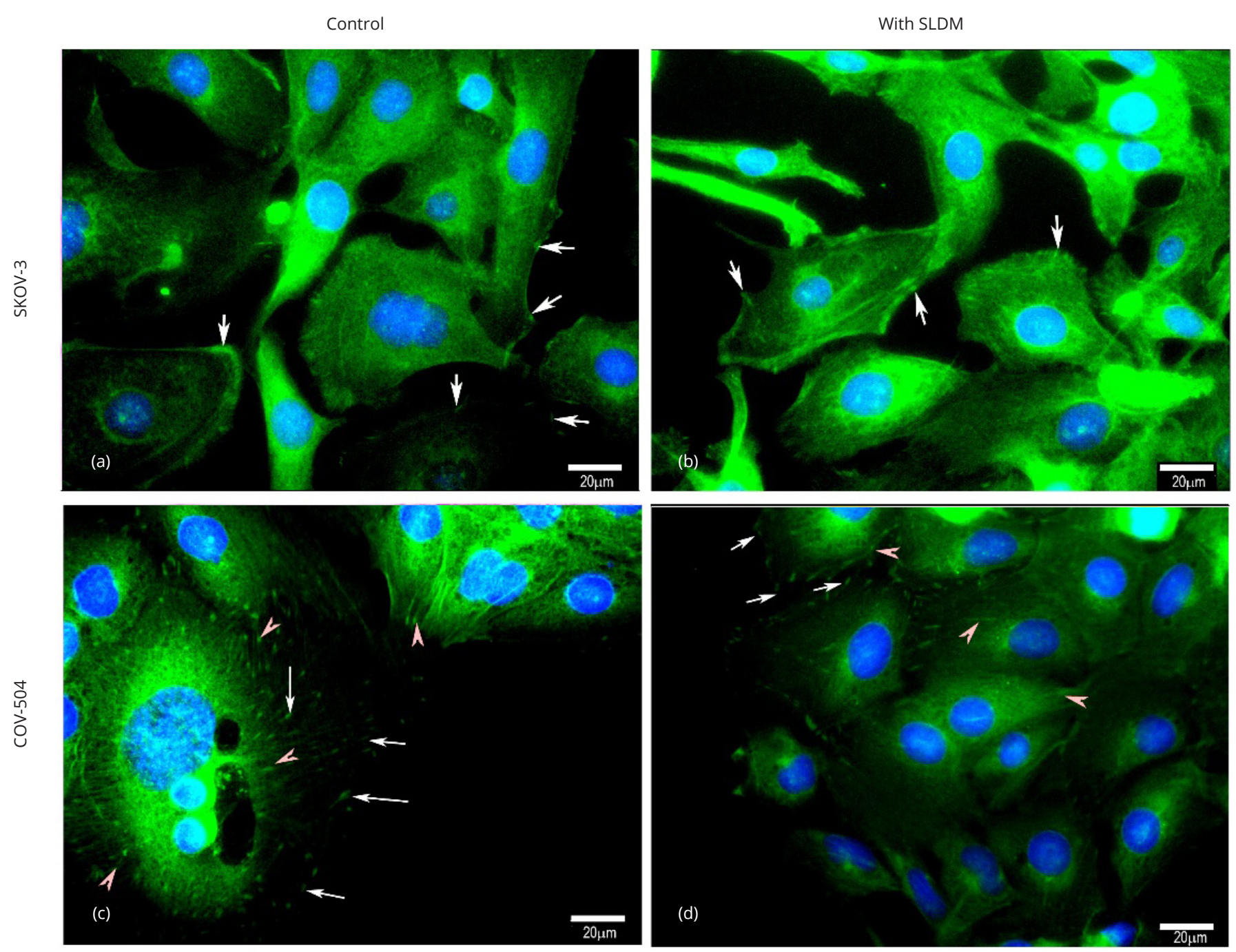

Figure 2a,b,c,d Paxillin immunofluorescence staining in ovarian cancer cells.

Paxillin staining of SKOV-3 cells (top panel) and COV-504 cells (bottom panel). In the untreated SKOV-3 cells (top left panel) paxillin staining generally appears to be diffuse in the cytoplasm with some small clusters (indicated by white arrows) appearing at the cell membrane. In SKOV-3 cells treated with SLDM (top right panel) in some of the cells staining appears to have clustered, potentially indicating focal adhesion complexes, at the cell membranes (white arrows). Within the control COV-504 cells paxillin staining indicative of focal adhesion clusters appeared through the cells (white arrows) and also appeared to align along the actin cytoskeleton (red arrows, bottom left panel). Treatment of COV-504 ovarian cancer cells with SLDM resulted in disruption and reduction of the paxillin stained clusters at the leading edges of the cells indicative of loss of focal adhesion clusters (white arrows, top and bottom right panels) with the staining appearing diffuse in the cytoplasm (red arrows, bottom right panel).

ShenLinLan resulted in a concentration dependent inhibition of cell adhesion and migration alone and in combination with an FAK inhibitor

Using an ECIS based method, we tested the potential effects of SLDM on ovarian cancer cell adhesion and migration. Previous work has demonstrated that SLDM does not have a toxic effect on ovarian cancer cells in vitro $[20,21]$.
ECIS modelling was used to qualitatively examine ovarian cancer cell adhesion and migration. SLDM inhibited ovarian cancer cell attachment and migration in a concentration dependent manner (Figure 3). Treating SKOV-3 or COV-504 cells with an FAK small inhibitor, PF537228 also appeared to result in a decrease in cellular attachment and migration. The combination treatment of increasing concentrations of SLDM and PF537228 resulted in a further decrease in ovarian cancer cell attachment and migration.

Qualitatively analysed, using ECIS modelling, treatment of COV-504 and SKOV-3 ovarian cancer cells with SLDM decreased their cell attachment (top panels) and cellular migration (bottom panels) as we previously reported [20]. This concentration dependent inhibition of cellular attachment (top panels) and migration (bottom panels) was further inhibited when ovarian cancer cells were treated with a combination of increasing SLDM concentrations and an FAK small inhibitor PF537228.

\section{Human ovarian tumours had highly expressed FAK and paxillin}

In our cohort of patients FAK and paxillin transcript levels were assessed using qPCR (Figure 4). FAK and paxillin transcripts were higher in samples from patients who had died from the disease (versus living) though this did not 

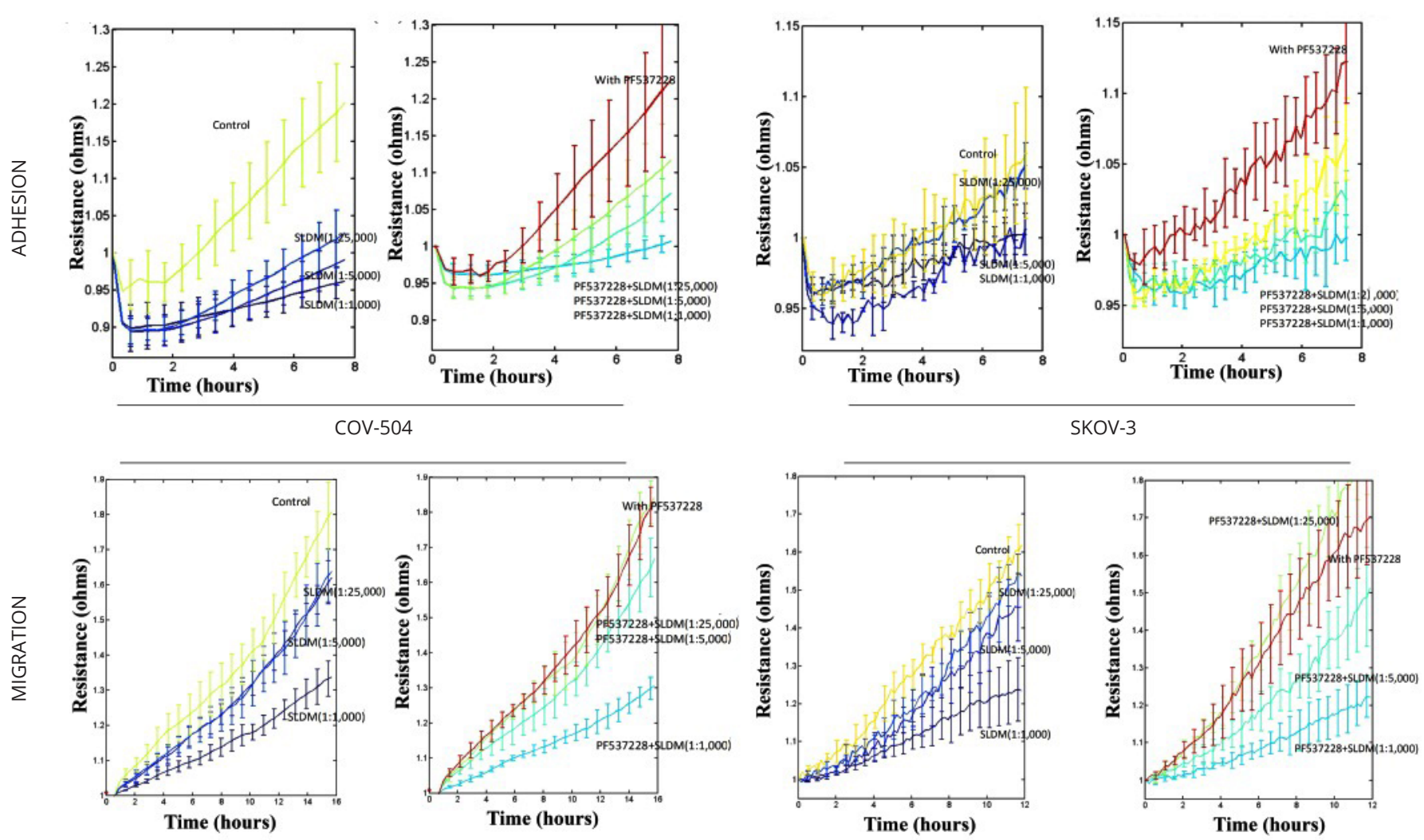

Figure 3 Inhibition of ovarian cancer cell adhesion and migration.

cross the statistical significance threshold. FAK transcript copy numbers were significantly increased in patients who had cancer related incidence (tumour recurrence or metastasis) compared to those patients who were incidence free $(p<0.05)$ (Figure $4 a)$. Increased paxillin transcript copies were also seen in patients with cancer related incidence (versus incidence free) however this did not reach statistical significance (Figure 4b). Ovarian cancer

(a)

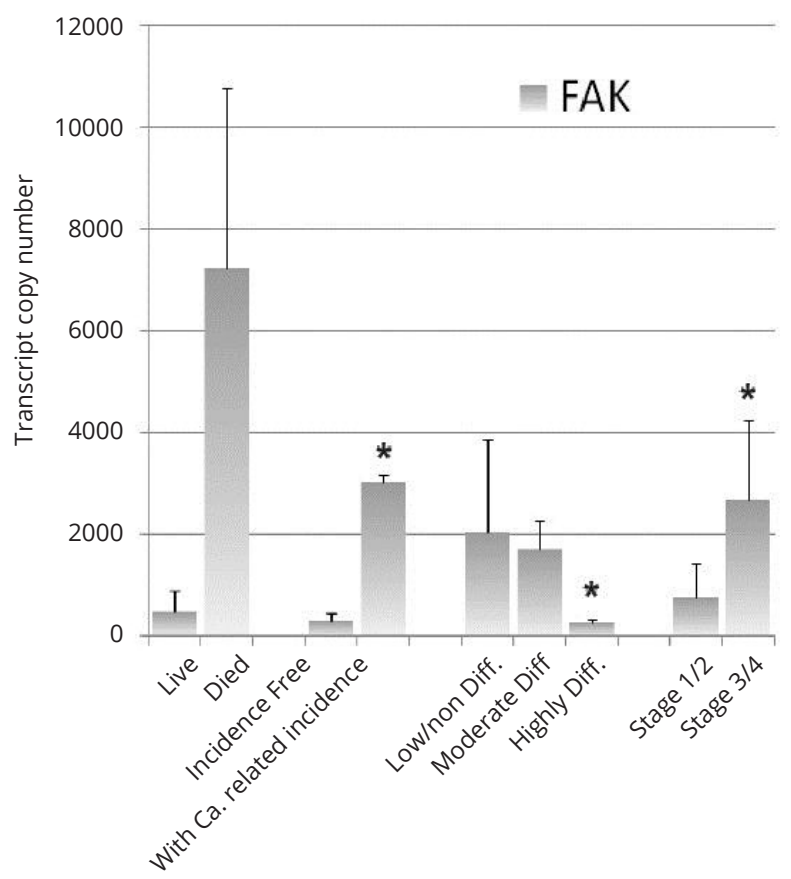

tumours which were classified as highly differentiated, had significantly lower transcript numbers of FAK and paxillin compared to those that had been classified as moderately or poorly differentiated tumours $(p<0.05)$. Patients who were scored with high grade tumours (III and IV) also showed significantly increased transcript copies of FAK and paxillin compared to those patients who were scored with lower grade tumours $(I$ and II) $(p<0.05)$.

(b)

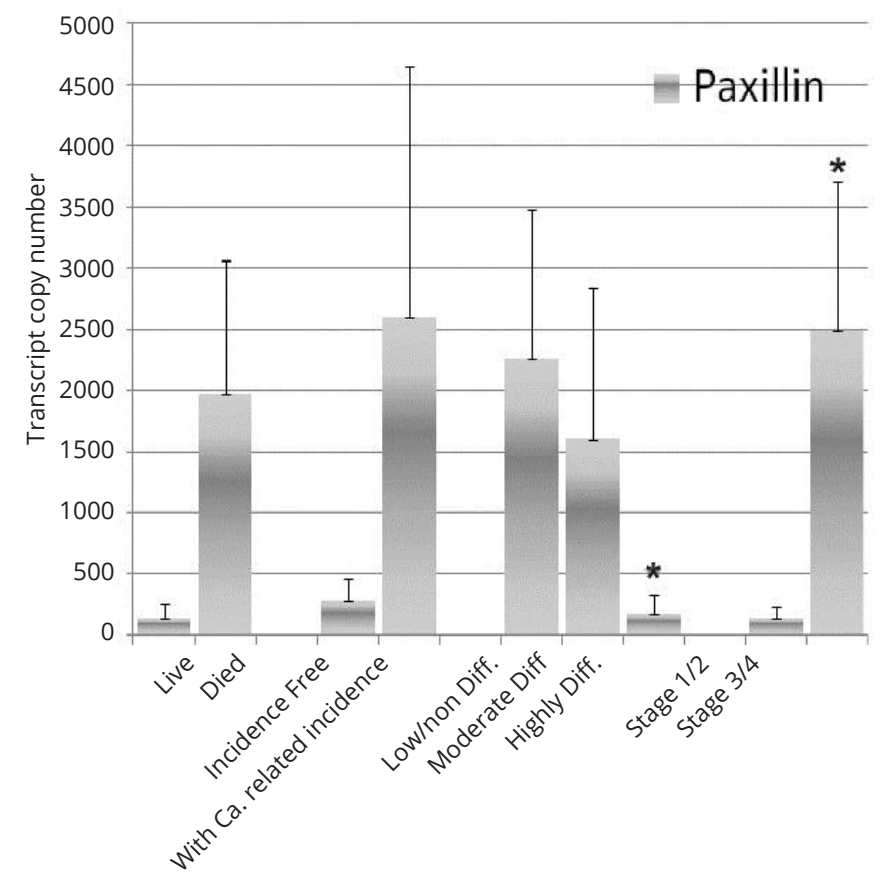

Figure 4 FAK and paxillin transcript levels in ovarian cancer tumours. 
FAK transcript copies were increased in those patients who had died of the disease, and were significantly increased in patients with cancer related incidence (tumour recurrence and/or metastasis) compared with those who remained incidence free and those patients who had higher grade tumours (III and IV) compared to those with lower grade tumours (I and II) $(p<0.05)(a)$. Significantly lower transcript copies of FAK were seen in patients with highly differentiated tumours compared with those tumours which were moderately or poorly differentiated $(p<0.05)$. Paxillin transcript levels were also increased in patients who died of the disease (vs those who survived) and in those patients with cancer related incidence (vs those who were incidence free) though these increases did not reach a statistical significance (b). Significantly lower transcript copies of paxillin were seen in patients with highly differentiated tumours compared with those tumours which were moderately or poorly differentiated $(p<0.05)$. Significantly increased paxillin transcript levels were seen in patients with higher grade tumours (III and IV) compared to those with lower grade tumours (I and II) $(p<0.05)$.

\section{Discussion}

Ovarian cancer remains a major clinical problem as it is generally detected in the latter stages of the disease, when it is more difficult to treat, and metastasis is more likely to have occurred. The FAK and paxillin pathways have been heavily linked to the metastatic process of solid tumours. Results from our clinical cohort suggest that ovarian cancer is similar to other solid tumours, including breast and lung, in which increased transcript copy numbers of FAK and paxillin are linked to poor prognosis [23-28]. Our clinical cohort isolated those groups of patients who had poorly differentiated tumours, and those patients who died from the disease and/or had associated incidence.

The data from our study also provides insight into the mechanisms by which SLDM may exert some of its therapeutic effects in the treatment of cancer. Through the use of the protein array we were able to identify changes in FAK and paxillin. Of greater interest was the identification of some key phosphorylation sites which were affected after treatment with SLDM.

Tyrosine phosphorylation (397) of FAK occurs in response to a range of stimuli, including integrin clustering and growth factors, and has been identified as key to FAK's intrinsic activity as well as providing a binding site for Src family kinases as well as other proteins [29, 30]. The decrease in autophosphorylation which was observed at FAK Y-397 is therefore of particular interest when taken into consideration with the observation by Tancioni et al. in which OPN, integrin $\beta_{5}$ and FAK control ovarian cancer spheroid growth [31]. Grisaru-Granovsky et al. [32] identified that pY397 FAK and phosphorylated PAR1 were upregulated in ovarian cancer tissue and that possible interactions contributed to ovarian cancer [32]. Further research has shown that ovarian cancer cells metastasise as spheroid clusters in which FAK signalling functions and its downstream effectors support tumour cell survival [33, 34].
Interestingly the phosphorylation of S-910 was also decreased, as seen from the antibody array data, suggesting that SLDM may exert its effects on FAK at more than one phosphorylation site and may potentially affect ERK [35]. The Kinexus data highlights that part of the actions observed with SLDM treatment are linked to key phosphorylation events in the FAK pathway in particular. The decreased autophosphorylation at the Y-397 site, given its links with integrin and growth factor interactions, is a key observation and this site has been the target of many FAK specific small inhibitors as well as combination therapies between the FAK small inhibitors and other treatment $[8,10]$.

SLDM has a significant impact on the behaviour of ovarian cancer cell adhesion and migration, though not cell growth $[20,21]$. Treatment with SLDM resulted in a concentration dependent inhibition of ovarian cell adhesion and migration, this was further evident when added in combination with a FAK small inhibitor. The expression of FAK has been demonstrated in a number of tumour types and has been linked to the clinical outcome of the patients. This link was well demonstrated in a meta-analysis, including over four thousands patients with varying tumour types, in that high FAK expression was highly associated with the overall survival [36]. The study has shown that this link to survival was highly significant for gastric cancer, liver cancer, ovarian and endometrial cancers and glioma. Ward et al. have also previously demonstrated that targeting FAK inhibits tumour progression and improves survival of the patients with ovarian cancer [33].

In a previous study exploring the potential mechanism(s) of action by which SLDM, the novel TCM derivative of YZXJ, exerts its anticancer effects we have reported that GSK-3 is also affected in vitro, and that combining SLDM with a GSK$3 \beta$ targeted small inhibitor also synergistically decreased cellular migration. This multi-targeted effect therefore makes it difficult, at this initial stage, to isolate which of these pathways has the greater influence on reducing ovarian cancer cell migration in vitro. Furthermore, cellular migration is linked to other key cancer traits such as angiogenesis. YZXJ has demonstrated anti-angiogenic effects on a variety of solid cancer cell types in vitro however as yet this property has not been investigated with treatment of SLDM.

Though SLDM appears to affect both SKOV-3 and COV504 cell adhesion and migration, the way in which these effects are achieved may differ in the different cell lines. Immunofluorescence staining of paxillin in this study showed that the different cell lines exhibited differing localisation of paxillin in the untreated controls. This in itself is interesting given that ovarian cancer is a heterogenous disease and SKOV-3 is derived from an epithelial adenocarcinoma and COV-504 is derived from the pleural effusion of an epithelial serous carcinoma. Therefore further investigation is required using both cell lines to investigate the possibility that differing components of SLDM affect various pathways, potentially through FAK, unique to the differing ovarian cancer cell molecular characteristics. 
Beyond the scope of this study, future investigation is further required to analyse the effects SLDM may have on other cancer cell traits, such as cell invasion, and the potential pathways implicated both at an in vitro and in vivo level. Beyond this, further bio-chemical analysis could allow for isolation of the exact chemical components within the SLDM which exert these effects and explore if its' anticancer effects are derived from a synergistic rather than single targeted approach.

Subsequent work is now needed to further understand how this medicine, through isolating the fundamental mechanisms of action, could benefit cancer patients, potentially through combination with chemotherapeutic agents, with further in vitro and in vivo studies.

\section{Conflict of Interest}

The authors declare no conflict of interest.

\section{Acknowledgement}

The authors wish to thank the Welsh National Research Network/ Ser Cymru and Cancer Research Wales for supporting their work.

\section{Author Contributions}

YW and WGJ conceived the research inception, YG, CW, HW, YG and WGJ conceived and designed the experiments; SO, FR and WGJ performed the experiments and analysed the data, YG, YY, JH, JC contributed to clinical cohort and clinical follow-up, HW, CW and YW contributed reagents and materials and their preparation; SO, WGJ and JL wrote the paper.

\section{References}

[1] Chen T, You Y, Jiang H, Wang ZZ. Epithelial-mesenchymal transition (EMT): A biological process in the development, stem cell differentiation and tumorigenesis. J Cell Physiol. 2017.

[2] Ata R, Antonescu CN. Integrins and cell metabolism: An intimate relationship impacting cancer. Int J Mol Sci. 2017; 18(1):189.

[3] Zhao X, Guan JL. Focal adhesion kinase and its signaling pathways in cell migration and angiogenesis. Adv Drug Deliv Rev. 2011; 63(8):610615.

[4] Sood AK, Coffin JE, Schneider GB, Fletcher MS, DeYoung BR, et al. Biological significance of focal adhesion kinase in ovarian cancer: Role in migration and invasion. Am J Pathol. 2004; 165(4):1087-1095.

[5] Luo M, Guan JL. Focal adhesion kinase: A prominent determinant in breast cancer initiation, progression and metastasis. Cancer Lett. 2010; 289(2):127-39.

[6] Ovarian cancer statistics. World cancer research fund international.

[7] Maringe C, Walters S, Butler J, Coleman MP, Hacker N, et al. Stage at diagnosis and ovarian cancer survival: Evidence from the international cancer benchmarking partnership. Gynecol Oncol. 2012; 127(1):7582.

[8] Golubovskaya VM. Targeting fak in human cancer: From finding to first clinical trials. Front Biosci (Landmark Ed). 2014; 19:687-706.

[9] Kanteti R, Batra SK, Lennon FE, Salgia R. Fak and paxillin, two potential targets in pancreatic cancer. Oncotarget. 2016; 7(21):31586-31601.

[10] O'Brien S, Golubovskaya VM, ConroyJ, Liu S, Wang D, et al. Fak inhibition with small molecule inhibitor y15 decreases viability, clonogenicity, and cell attachment in thyroid cancer cell lines and synergizes with targeted therapeutics. Oncotarget. 2014; 5(17):7945-7959.

[11] Deakin NO, Pignatelli J, Turner CE. Diverse roles for the paxillin family of proteins in cancer. Genes Cancer. 2012; 3(5-6):362-370.

[12] Jiang WG, Ye L, Ji K, Frewer N, Ji J, et al. Inhibitory effects of yangzheng xiaoji on angiogenesis and the role of the focal adhesion kinase pathway. Int J Oncol. 2012; 41(5):1635-1642.
[13] Jiang WG, Ye L, Ruge F, Owen S, Martin T, et al. Yangzheng xiaoji exerts anti-tumour growth effects by antagonising the effects of hgf and its receptor, cmet, in human lung cancer cells. J Transl Med. 2015; 13:280.

[14] Jiang WG, Ye L, Ji K, Ruge F, Wu Y, et al. Antitumour effects of Yangzheng Xiaoji in human osteosarcoma: The pivotal role of focal adhesion kinase signalling. Oncol Rep. 2013; 30(3):1405-1413.

[15] Owen S, Zhao H, Dart A, Wang Y, Ruge F, et al. Heat shock protein 27 is a potential indicator for response to Yangzheng Xiaoji and chemotherapy agents in cancer cells. Int J Oncol. 2016; 49(5):1839-1847.

[16] Ye L, Ji K, Frewer N, Ji J, Jiang WG. Impact of yangzheng xiaoji on the adhesion and migration of human cancer cells: The role of the akt signalling pathway. Anticancer Res. 2012; 32(7):2537-2543.

[17] Xingjun Cui WM, Xuejie Bi. Effect of Yangzheng Xiaoji capsule on cellular immune function in patients with advanced gastric cancer chemotherapy. Chinese Journal of Difficult and Complicated Cases. 2011; 10:703-704.

[18] Xue Kan SF, Ji Jiafu. Meta-anlaysis of the safety of Yangzheng Xiaoji capsule for the treatment of cancer and precancerosis. Chinese Journal Clinical Oncology. 2013; 40:1318-1323.

[19] Chen J, Wu X. Shenling/an in the treatment of chronic hepatitis-b, a clinical investigation of 30 patients cohort. LiaoNian Journal of Traditional Chinese Medicine. 2006; 33:60.

[20] Nazarenko IA, Bhatnagar SK, Hohman RJ. A closed tube format for amplification and detection of DNA based on energy transfer. Nucleic Acid Res. 1997; 25(1):2516-2521.

[21] Jiang WG, Watkins G, Lane J, Cunnick GH, Douglas-Jones A, et al. Prognostic value of rho gtpases and rho guanine nucleotide dissociation inhibitors in human breast cancers. Clin Cancer Res. 2003; 9(17):6432-6440.

[22] Davies SR, Davies ML, Sanders A, Parr C, Torkington J, et al. Differential expression of the ccn family member wisp-1, wisp-2 and wisp-3 in human colorectal cancer and the prognostic implications. Int J Oncol. 2010; 36(5):1129-1136.

[23] Ji HF, Pang D, Fu SB, Jin Y, Yao L, et al. Overexpression of focal adhesion kinase correlates with increased lymph node metastasis and poor prognosis in non-small-cell lung cancer. J Cancer Res Clin Oncol. 2013; 139(3):429-435.

[24] Miyazaki T, Kato $H$, Nakajima $M$, Sohda $M$, Fukai $Y$, et al. Fak overexpression is correlated with tumour invasiveness and lymph node metastasis in oesophageal squamous cell carcinoma. Br J Cancer. 2003; 89(1):140-145.

[25] Yom CK, Noh DY, Kim WH, Kim HS. Clinical significance of high focal adhesion kinase gene copy number and overexpression in invasive breast cancer. Breast Cancer Res Treat. 2011; 128(3):647-655.

[26] Zeng XQ, Li N, Ma LL, Tseng YJ, Zhao NQ, et al. Prognostic value of focal adhesion kinase (FAK) in human solid carcinomas: A meta-analysis. PLoS One. 2016; 11(9):e0162666.

[27] Du C, Wang X, Zhang J, Liu X, Zhu J, et al. Paxillin is positively correlated with the clinicopathological factors of colorectal cancer, and knockdown of paxillin improves sensitivity to cetuximab in colorectal cancer cells. Oncol Rep. 2016; 35(1):409-417.

[28] Xiao LJ, Zhao EH, Zhao S, Zheng X, Zheng HC, et al. Paxillin expression is closely linked to the pathogenesis, progression and prognosis of gastric carcinomas. Oncol Lett. 2014; 7(1):189-194.

[29] Hanks SK, Ryzhova L, Shin NY, Brábek J. Focal adhesion kinase signaling activities and their implications in the control of cell survival and motility. Front Biosci. 2003; 8:d982-996.

[30] Schaller MD, Hildebrand JD, Shannon JD, Fox JW, Vines RR. Autophosphorylation of the focal adhesion kinase, pp125fak, directs sh2-dependent binding of pp60src. Mol Cell Biol. 1994; 14(3):16801688.

[31] Tancioni I, Uryu S, Sulzmaier FJ, Shah NR, Lawson C, et al. Fak inhibition disrupts a beta5 integrin signaling axis controlling anchorageindependent ovarian carcinoma growth. Mol Cancer Ther. 2014; 13(8):2050-2061.

[32] Grisaru-Granovsky S, Salah Z, Maoz M, Pruss D, Beller U, et al. Differential expression of protease activated receptor 1 (par1) and py397fak in benign and malignant human ovarian tissue samples. Int J Cancer. 2005; 113(3):372-378.

[33] Ward KK, Tancioni I, Lawson C, Miller NL, Jean C, et al. Inhibition of focal adhesion kinase (FAK) activity prevents anchorage-independent ovarian carcinoma cell growth and tumor progression. Clin Exp Metastasis. 2013; 30(5):579-594.

[34] Seton-Rogers S. Ovarian cancer: Driving force. Nat Rev Cancer. 2011; 11(8):538-539.

[35] Villa-Moruzzi E. Tyrosine phosphatases in the HER2-directed motility of ovarian cancer cells: Involvement of PTPN12, ERK5 and FAK. Anal Cell Pathol (Amst). 2011; 34(3):101-112.

[36] Zheng Y, Lu Z. Paradoxical roles of FAK in tumor cell migration and metastasis. Cell Cycle. 2009; 8(21):3474-3479. 